\title{
Real-Time Sleep Apnea Detection by Classifier Combination
}

\author{
Baile Xie, Student Member, IEEE, and Hlaing Minn, Senior Member, IEEE
}

\begin{abstract}
To find an efficient and valid alternative of polysomnography (PSG), this paper investigates real-time sleep apnea and hypopnea syndrome (SAHS) detection based on electrocardiograph (ECG) and saturation of peripheral oxygen $\left(\mathrm{SpO}_{2}\right)$ signals, individually and in combination. We include ten machinelearning algorithms in our classification experiment. It is shown that our proposed $\mathrm{SpO}_{2}$ features outperform the ECG features in terms of diagnostic ability. More importantly, we propose classifier combination to further enhance the classification performance by harnessing the complementary information provided by individual classifiers. With our selected $\mathrm{SpO}_{2}$ and ECG features, the classifier combination using AdaBoost with Decision Stump, Bagging with REPTree, and either $k N N$ or Decision Table achieves sensitivity, specificity, and accuracy all around $82 \%$ for a minute-based realtime SAHS detection over 25 sleep-disordered-breathing suspects' full overnight recordings.
\end{abstract}

Index Terms-Classifier combination, electrocardiograph (ECG), feature selection, hypopnea, machine learning, saturation of peripheral oxygen $\left(\mathrm{SpO}_{2}\right)$, sleep apnea.

\section{INTRODUCTION}

$\mathbf{S}$ LEEP apnea and hypopnea syndrome (SAHS) is a common sleep disorder which is characterized by abnormal breath pause or reduction during sleep. It is estimated to affect $2 \%$ of middle-aged women and $4 \%$ of middle-aged men [1]. Sleep apnea is treatable; however, about $90 \%$ of sufferers go unidentified and hence untreated [2]. They experience daytime sleepiness and fatigue which can escalate to traffic accidents, depression, and memory loss. Moreover, untreated SAHS can also relate to ischemic heart disease, cardiovascular disfunction, and stroke [3], [4]. A common definition of apnea involves a cessation of airflow for at least $10 \mathrm{~s}$ while hypopnea is defined as a minimum 10-s airflow reduction with either a blood oxygen desaturation of $4 \%$ or a neurological arousal [5]. Currently, polysomnography (PSG) is considered as the standard method for SAHS diagnosis. Nevertheless, PSG requires SAHS suspects to sleep in a sleep laboratory over one or two nights, with attended technicians. During the overnight sleep, a variety of sensors and wires are attached to the suspect's body. The recorded signals are then analyzed by sleep specialists for final diagnosis. The discomfort, inconvenience, and expensiveness of PSG set a barrier from its prevalence among public. Therefore, a

Manuscript received April 21, 2011; revised September 23, 2011 and January 19, 2012; accepted February 9, 2012. Date of publication February 16, 2012; date of current version May 4, 2012.

The authors are with the Department of Electrical Engineering, University of Texas at Dallas, Richardson, TX 75080 USA (e-mail: baile.xie@utdallas.edu; hlaing.minn@utdallas.edu).

Digital Object Identifier 10.1109/TITB.2012.2188299 readily available, relatively inexpensive, and reliable diagnosis alternative is desirable for public use.

In the past decade, several methods have been proposed as PSG alternatives for SAHS detection. For instance, detections have been developed based on questionnaires [6], snoring [7], electrocardiograph (ECG) [8]-[12], and pulse oximetry [5], [12]-[21]. Among them, ECG and saturation of oxygen measured by pulse oximeter $\left(\mathrm{SpO}_{2}\right)$ are the two most extensively studied signals.

Specifically, based on the ECG signal alone, various frequency and time domains as well as ECG morphology features have been developed. For example, McNames and Fraser [8] found that the heart rate (HR), the S-pulse amplitude, and the pulse energy of the ECG signal are informative for SAHS detection. Raymond et al. [9] analyzed the power spectral features of the ECG-derived respiration (EDR) signal via wavelet transform in addition to other features from the RR interval tachogram. Shinar et al. [10] extracted features from the changes of ECG signal's QRS complex and spectral abnormalities of heart rate variability (HRV).

$\mathrm{SpO}_{2}$ is the percentage $(\%)$ of hemoglobin in the blood that is saturated with oxygen recorded by a pulse oximeter. Some commonly used $\mathrm{SpO}_{2}$ features are the accumulative time (TSA) spent below a certain saturation level [5], [14], the oxygen desaturation index (ODI, the number of oxyhemoglobin desaturation below a certain threshold) [15], and the saturation variability index (Delta index) [5], [13], [14]. Later on, several nonlinear parameters such as approximate entropy (ApEnt) [16], central tendency measure (CTM), and Lempel-Ziv complexity (LZCom) [17] were also applied to sleep apnea detection. In the realm of spectral-domain features, Zamarrón et al. [18] studied the periodogram of the $\mathrm{SpO}_{2}$ signal and selected four indices related to the period $30-70 \mathrm{~s}$ for detection purpose. However, all the aforementioned methods rely on the entire overnight $\mathrm{SpO}_{2}$ records, resulting in a delayed offline analysis and diagnosis.

In order to obtain a real-time SAHS monitoring and diagnosis, some pioneering works have emerged. Oliver and Flores-Mangas [19] implemented a real-time detection system with oximetry but unfortunately lacked a performance comparison with the standard PSG. Heneghan et al. [12] adopted both ECG and $\mathrm{SpO}_{2}$ signals to estimate the apnea plus hypopnea index (AHI) based on an epoch-by-epoch detection. Most recently, Burgos et al. [20] and Bsoul et al. [11] have implemented a systematic real-time SAHS detection based on $\mathrm{SpO}_{2}$ alone and ECG alone, respectively.

In this paper, we focus on real-time sleep apnea/hypopnea detection based on 1-min segments of ECG and $\mathrm{SpO}_{2}$ signals. While following the comprehensive ECG features set in [11], we 


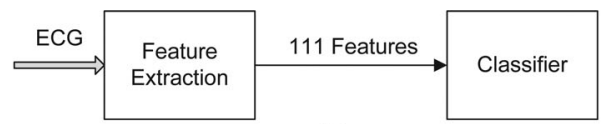

(a)

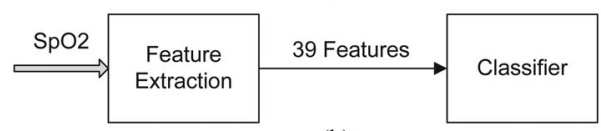

(b)

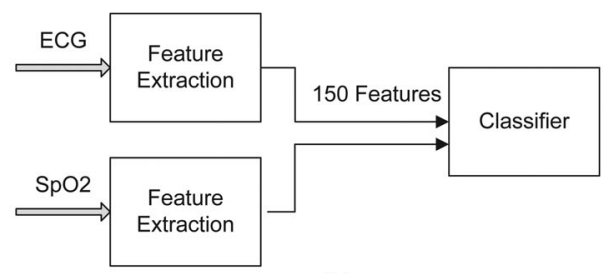

(c)

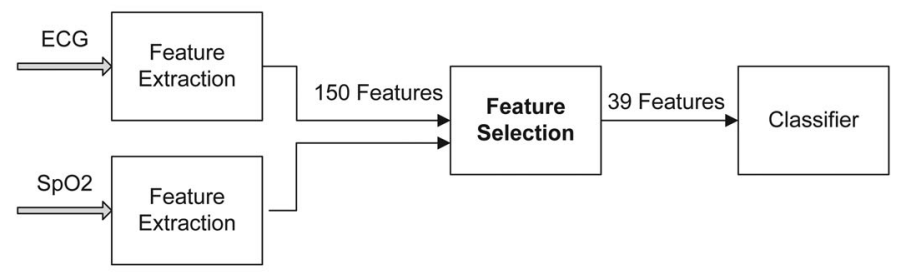

(d)

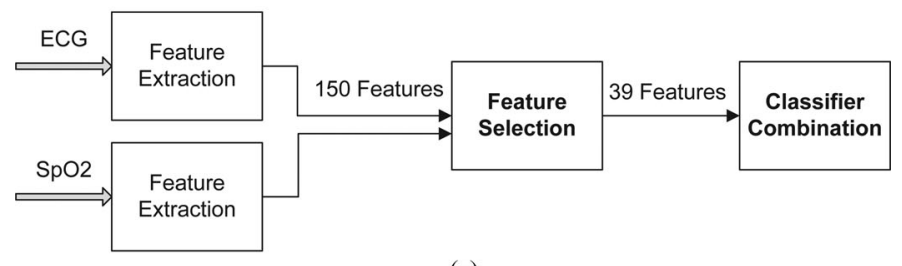

(e)

Fig. 1. Different strategies of SAHS detection. (a) SAHS detection based on the ECG signal only. (b) SAHS detection based on the $\mathrm{SpO}_{2}$ signal only. (c) SAHS detection based on both ECG and $\mathrm{SpO}_{2}$ signals. (d) SAHS detection based on ECG and $\mathrm{SpO}_{2}$ signals with feature selection. (e) SAHS detection based on selected ECG and $\mathrm{SpO}_{2}$ features with classifier combination.

develop an $\mathrm{SpO}_{2}$ feature set of 39 features. The different strategies of SAHS detection that we explore can be found in Fig. 1. To begin with, investigations of individual diagnostic abilities of the ECG and $\mathrm{SpO}_{2}$ features are carried out and compared via an experiment of ten different machine-learning algorithms. Costsensitive classification is included to enhance the sensitivity. Additionally, the classification experiment extends to involving a full set of 150 features fused by both $\mathrm{ECG}$ and $\mathrm{SpO}_{2}$ features. In contrast to feature selection based on individual evaluation of each feature as used in existing works [11], [20], [21], we utilize a subset feature selection strategy that accounts not only the prediction ability of each feature, but also the redundancy among features. The idea of cost-sensitive weighting is also incorporated in the feature selection process to favor highly predictive features. Most importantly, with investigation of the performances of individual classifiers, we introduce classifier combination to further enhance detection performance by harnessing the potentially complementary information provided by individual classifiers.

The rest of this paper is organized as follows. In Section II, we describe the database and the methods used in our experiment,
TABLE I

Physiological Properties of SubJeCts IN UCD Database

\begin{tabular}{|c|c|c|}
\hline & Mean Value & Range \\
\hline Age & $50 \pm 10$ years & $28-68$ years \\
\hline Body Mass Index & $31.6 \pm 4.0 \mathrm{~kg} / \mathrm{m}^{2}$ & $25.1-42.5 \mathrm{~kg} / \mathrm{m}^{2}$ \\
\hline AHI & $24.1 \pm 20.3$ & $1.7-90.9$ \\
\hline
\end{tabular}

including signal processing, feature extraction, feature selection, and classifier combination. Section III shows the results of different classification strategies. In Section IV, we discuss the performances among different feature sets and classification schemes, and compare our results with those of other research groups. Finally, Section V concludes this paper.

\section{Materials AND MEtHODS}

\section{A. Database}

Throughout this paper, we use the St. Vincent's University Hospital/University College Dublin Sleep Apnea Database (UCD database) [22] available online from PhysioNet [23] which provides a variety of physiological signals for biomedical research. The availability of the UCD database offers easy validation and assessment of our approach. Twenty-five (21 males and 4 females) sleep-disordered-breathing suspects' full overnight PSG recordings are recorded in the database. Each recording contains 5.9- to 7.7-h ECG and $\mathrm{SpO}_{2}$ signals as well as an annotation file with detailed onset time and duration of every apnea/hypopnea event. Polysomnograms were obtained using the Jaeger-Toennies system (Erich Jaeger GmbH, Germany). In particular, the ECG signal was recorded via a modified lead V2 and the $\mathrm{SpO}_{2}$ signal was recorded using a finger pulse oximeter. General physiological properties of the subjects are listed in Table I while a more detailed description can be found online [22].

\section{B. Signal Processing and Feature Extraction}

The ECG and $\mathrm{SpO}_{2}$ signals in the database are originally sampled by 128 and $8 \mathrm{~Hz}$, respectively. In our experiment, both signals are segmented into 1-min episodes for signal processing and the detection/classification results are returned minute by minute. Accordingly, the annotations need to be modified to give minute-based references. Given that the apnea/hypopnea happens with a minimum of 10-s airflow change, in case the events are across two adjacent segments, we label a single minute as "Apnea" (apneic) if it contains at least 5 consecutive seconds of apnea/hypopnea events; otherwise, this minute is annotated as "No apnea" (normal). We use MATLAB for signal processing and feature extraction.

Recently, Bsoul et al. [11] have proposed a quite comprehensive ECG feature set which contains $111 \mathrm{HRV}$ - and EDR-based features in both time and spectral domains. In this paper, we employ this feature set for the ECG signal and mainly focus on feature designs of the $\mathrm{SpO}_{2}$ signal due to its strong reflection of arterial oxygen saturation on the airflow fluctuation.

To begin with, the $\mathrm{SpO}_{2}$ signal is downsampled at $1 \mathrm{~Hz}$. Any $\mathrm{SpO}_{2}$ values less than 50 are considered as artifacts and hence 
removed from analysis (totally about $137 \mathrm{~min} ; 1.3 \%$ of data are removed).

Basic statistics such as the minimum, mean, variance, and correlation coefficient of $\mathrm{SpO}_{2}$ samples within each segment are first calculated and denoted as Smini, Smean, Svari, and CorreC, respectively. Then using Smean as a baseline, the number of zero crossing points of each segment is counted as feature NumZC. Within each segment, via linear regression, a regression line is fitted. The Slope, its absolute value (AbSlope), and Bias of the regression line are measured as three additional features.

Delta index is viewed as a valid parameter for overnight SAHS detection [5], [13], [14]. In our real-time processing, the mean value of the $\mathrm{SpO}_{2}$ signal over every 12-s interval is first computed and the Delta index (Dmean) is derived as the one-min average of the absolute differences between two successive mean values.

The nonlinear methods such as ApEnt, CTM, and LZCom, which measure the regularity, variability, and complexity of a time series, have been applied to SAHS detection based on overnight $\mathrm{SpO}_{2}$ signals [16], [17]. These three features can also be easily calculated segmentwise. In particular, for ApEnt, we choose the optimal run length of 1 and tolerance window of 0.25 times the standard deviation of each epoch data, as suggested in [16]. The CTM is calculated by selecting a radius with respect to (w.r.t.) the origin of a second-order difference plot and counting the number of points which fall within the radius [17]. We choose radii of $0.25,0.5,0.75$, and 1 , corresponding to features CTM25, CTM50, CTM75, and CTM100, respectively.

Besides three ODI indices (odi2, odi3, odi4) in [20], we adopt a more general definition of ODI indices as in [15]. We set the baseline as the mean of the top $20 \%$ of the $\mathrm{SpO}_{2}$ data within 1 min. The ODI index ODIxy counts the occurrences that $\mathrm{SpO}_{2}$ samples drop at least $x$ below the baseline and last at least $y$ s. In our experiment, $x \in\{2,3,4,5\}$ and $y \in\{1,3,5\}$. For example, ODI21 presents the number of times when the $\mathrm{SpO}_{2}$ level declines at least 2 below the baseline and lasts at least $1 \mathrm{~s}$. Additionally, we count the total number of $\mathrm{SpO}_{2}$ samples which fall at least 2, 3, 4, and 5 below the baseline, contributing another four features: ODIS2, ODIS3, ODIS4, and ODIS5.

Finally, we consider five TSA indices (tsa95, tsa90, tsa85, tsa80, tsa70) indicating the accumulative time that the $\mathrm{SpO}_{2}$ level stays below 95, 90, 85, 80, and 70, respectively.

As a result, totally, a set of $39 \mathrm{SpO}_{2}$ features is built. ${ }^{1}$ The aforementioned $\mathrm{SpO}_{2}$ features and their descriptions are summarized in Table II for ease of reference.

\section{Classification}

In the classification phase, we employ an open-source machine-learning software, WEKA [24], as the major tool to assess the performances of the aforesaid feature sets. Ten classifiers are included in our experiments. Specifically, support vector machine (SVM) [25] maps data into a high-dimensional space and constructs a hyperplane to separate them. $k$-nearest

\footnotetext{
${ }^{1}$ We mainly focus on the time-domain features in our real-time processing since the apnea/hypopnea event can last as long as $120 \mathrm{~s}$ [19], which exceeds the epoch length. Part of the $\mathrm{SpO}_{2}$ features is used in our conference paper [21].
}

TABLE II

$\mathrm{SPO}_{2}$ FEATURES AND THEIR DESCRIPTIONS

\begin{tabular}{|c|c|}
\hline Feature Name & Description \\
\hline Smini & Minimum of 1-minute $\mathrm{SpO}_{2}$ samples \\
\hline Smean & Mean value of 1-minute $\mathrm{SpO}_{2}$ samples \\
\hline Svari & Variance of 1-minute $\mathrm{SpO}_{2}$ samples \\
\hline CorreC & Correlation coefficient of 1-minute $\mathrm{SpO}_{2}$ samples \\
\hline NumZC & $\begin{array}{l}\text { Number of zero crossing points within 1-minute } \\
\mathrm{SpO}_{2} \text { samples, using Smean as baseline }\end{array}$ \\
\hline Slope & $\begin{array}{l}\text { Slope of the regression line fitted for 1-minute } \mathrm{SpO}_{2} \\
\text { samples }\end{array}$ \\
\hline AbsSlope & Absolute value of Slope \\
\hline Bias & $\begin{array}{l}\text { Bias of the regression line fitted for 1-minute } \mathrm{SpO}_{2} \\
\text { samples }\end{array}$ \\
\hline Dmean & $\begin{array}{l}\text { Delta index: 1-minute average of the absolute differ- } \\
\text { ences between two successive mean values of } \mathrm{SpO}_{2} \\
\text { signal over } 12 \text {-second intervals }\end{array}$ \\
\hline ApEnt & $\begin{array}{l}\text { Approximate entropy [16] with run length of } 1 \text { and } \\
\text { tolerance window of } 0.25 \sqrt{\text { Svari }}\end{array}$ \\
\hline LZCom & Lempel-Ziv complexity [17] \\
\hline $\begin{array}{l}\text { CTM25, } \\
\text { CTM50, } \\
\text { CTM75, } \\
\text { CTM100 }\end{array}$ & $\begin{array}{l}\text { Central tendency measure [17] with radius } \\
0.25,0.50,0.75,1.00 \text { (calculated by selecting } \\
\text { a radius w.r.t. the origin of a second-order difference } \\
\text { plot and counting the number of points that fall } \\
\text { within the radius) }\end{array}$ \\
\hline odi2, odi3, odi4 & Oxygen desaturation index defined in [20] \\
\hline $\begin{array}{l}\text { ODIxy, } x \\
\{2,3,4,5\} \\
y \in\{1,3,5\}\end{array}$ & $\begin{array}{l}\text { The number of occurrences that } \mathrm{SpO}_{2} \text { level declines } \\
\text { at least } x \text { below the baseline and lasts at least } y \\
\text { seconds (The baseline is the mean value of the top } \\
20 \% \text { of the } \mathrm{SpO}_{2} \text { data within } 1 \text { minute.) }\end{array}$ \\
\hline $\begin{array}{l}\text { ODIS2, ODIS3, } \\
\text { ODIS4, ODIS5 }\end{array}$ & $\begin{array}{l}\text { The total number of } \mathrm{SpO}_{2} \text { samples that fall at least } \\
2,3,4,5 \text { below the baseline (The baseline is the } \\
\text { mean value of the top } 20 \% \text { of the } \mathrm{SpO}_{2} \text { data within } \\
1 \text { minute.) }\end{array}$ \\
\hline $\begin{array}{ll}\text { tsa } 70, & \text { tsa80, } \\
\text { tsa } 85, & \text { tsa90, } \\
\text { tsa95 } & \end{array}$ & $\begin{array}{l}\text { Accumulative time that } \mathrm{SpO}_{2} \text { level stays below } \\
70,80,85,90,95\end{array}$ \\
\hline
\end{tabular}

neighbor $(k N N)$ [26] assigns the data the most common class among their $k$ closest neighbors. Decision Table classifier [27] builds a simple hypothesis space represented by a decision table and uses it for classification. Multilayer perceptron (MLP) [28] is an artificial neural network consisting of multiple layers of nodes; it models data pattern during training and follows the pattern to classify the testing data. Decision tree partitions data into different groups recursively. Several popular decision trees and their evolved versions are included, such as $C 4.5$ tree [29], reduced-error pruning tree (REPTree), and functional trees (FT trees) [30]. We also take into consideration several meta-algorithms which are used in conjunction with other (simple) classifiers to reduce prediction errors. In particular, Adaptive Boosting (AdaBoost) with Decision Stump [31], Bagging with REPTree [32], and Bagging with Alternating Decision Tree (ADTree) [33] are included in our experiment.

Throughout the experiment, the default parameter settings of each classifier are kept, except that for SVM; we normalize all the features into the $[0,1]$ region since SVM is sensitive to the dynamic ranges of the features. And for $k N N$ classifier, we choose $k=5$ and apply an inverse distance weight function.

To assess the classification performance, we use sensitivity, specificity, and accuracy as evaluation metrics. Their definitions are as follows:

$$
\text { sensitivity }=\frac{\mathrm{TP}}{\mathrm{TP}+\mathrm{FN}}
$$




$$
\begin{aligned}
\text { specificity } & =\frac{\mathrm{TN}}{\mathrm{TN}+\mathrm{FP}} \\
\text { accuracy } & =\frac{\mathrm{TP}+\mathrm{TN}}{\mathrm{P}+\mathrm{N}}
\end{aligned}
$$

where true positive (TP) and true negative (TN) refer to the number of correctly detected apneic epochs and normal epochs, respectively, whereas false positive (FP) and false negative (FN) stand for the number of miss-identified apneic and normal epochs, respectively. $P / N$ represents the total number of epochs with/without apneic events. In other words, the sensitivity reflects the ability to correctly detect the apneic epochs; specificity conveys the ability to distinguish the normal epochs. A tradeoff between the two usually exists in practice. In terms of SAHS detection problem, we are more interested in high sensitivity algorithms which reduce the risk of missing the apnea/hypopnea events that do pose threats to the patients. Therefore, we also carry out cost-sensitive classifications by imposing a cost matrix on the above ten classifiers, to penalize the FN errors more than the FP errors, and aim for a higher sensitivity.

As can be seen in Fig. 1, we first carry out experiments to assess and compare the individual diagnostic abilities of ECG and $\mathrm{SpO}_{2}$ features. The $111 \mathrm{ECG}$ features and $39 \mathrm{SpO}_{2}$ features are fed into the ten classifiers, respectively [see Fig. 1(a) and (b)]. In the next experiment, we fuse the two feature sets together and perform classification with a full set of 150 features [see Fig. 1(c)]. The experiments include both even cost (where the FN and FP are evenly weighted) and cost-sensitive classifications.

Note that all the experiment results are based on tenfold cross validation of the entire database. In particular, the entire dataset is evenly divided into ten folds; each time, one fold is left out for testing while the other nine folds are used for training. The testing results are averaged over ten folds and then returned as the final cross-validation results.

\section{Feature Selection}

Feature selection is regarded as a classic method to prevent overfitting by eliminating redundant or even detrimental features. In a real-time detection scenario, it is also an effective way to reduce the computational load by requiring less signal processing in feature extraction, to lower the rate of data transmission and energy consumption, and more importantly, to shorten the time required for model building during the training process.

Feature selection can be done by either investigating the value of each features, as done in [11], [20], and [21], or using a subset evaluator to assess the merit of a group of features. With the former method, no interinformation among features can be concluded, whereas the latter strategy can explore the correlation among features. Therefore, we employ the latter subset feature selection to further reduce redundancy.

In the interest of high sensitivity, we also use a cost-sensitive subset evaluator for feature selection. Cost-sensitive subset evaluator is a meta subset evaluator which requires a base subset evaluator and a cost matrix. In our case, the same cost matrix used in cost-sensitive classification is applied, and thus, the features with higher sensitivity are preferred. In terms of the base subset evaluator, correlation-based feature subset selection (CfsSubsetEval) [34] is employed independently of classifiers. CfsSubsetEval evaluates the merit of a subset of features by considering the individual predictive ability of each feature as well as the redundancy among them. To be specific, the merit of a subset $S$ is calculated as [34]

$$
M_{S}=\frac{k \overline{r_{\mathrm{cf}}}}{\sqrt{\left(k+k(k-1) \overline{r_{\mathrm{ff}}}\right)}}
$$

where $k$ is the number of features in $S$; $\overline{r_{\mathrm{cf}}}$ is the average classfeature correlation while $\overline{r_{\mathrm{ff}}}$ is the average feature-feature intercorrelation. As a result, a subset of features which are highly correlated with the class (apneic or normal) while having low intercorrelation among the features is preferred. In our experiment, feature selection is performed on the full feature set which consists of $111 \mathrm{ECG}$ and $39 \mathrm{SpO}_{2}$ features.

\section{E. Classifier Combination}

Naturally, one would want satisfactory results in all sensitivity, specificity, and accuracy. However, this ideal result does not always happen given a single classifier. Some classifiers provide high sensitivity but low specificity while others perform the opposite way. The gap between the two metrics can be large. To strike a balance, classifier combination is proposed as a potential solution.

Classifier combination has been proved to be a powerful method to improve the classification performance in many fields [35]. Inspired by the fact that the misclassified instances of individual classifiers do not necessarily overlap, different classifiers may offer different perspectives in decision making. Making use of those complementary information by classifier combination could further improve the performance.

In our combination experiment, we choose three individual classifiers to form a group of experts. Each member classifier predicts the class of every epoch and records the probabilities associated with both classes (apneic and normal). Since the prior class distribution of a database is usually unaware beforehand, an equiprobable prior is a common assumption. Based on the predicted classes and their corresponding probabilities, we explore four classifier combination schemes as described next.

1) MaxProbability (MP): This scheme assigns the predicted class as the one with the maximum probability among all classifiers and classes:

$$
C_{p}=\arg \max _{\{C+, C-\}} \max _{i}\left\{P_{i}(C+), P_{i}(C-)\right\}
$$

where $C_{p}$ is the final predicted class after combination and $i$ is the classifier index. $P_{i}(C+)$ and $P_{i}(C-)$ are the probabilities that classifier $i$ predicts the epoch to be positive (apneic) and negative (normal), respectively.

2) Average Probability $(A P)$ : In this rule, the probabilities of the positive/negative class are summed over all classifiers (can be viewed as the arithmetic mean criterion). The class with 
TABLE III

ClassificATION Results by Using EITHER ECG OR $\mathrm{SPO}_{2}$ FeATURE SET

\begin{tabular}{|c|c|c|c|c|c|c|c|c|c|c|c|c|}
\hline & \multicolumn{6}{|c|}{ Even Cost } & \multicolumn{6}{|c|}{ Cost Sensitive 3} \\
\hline & Sensit & ity $(\%)$ & Specif & ty(\%) & Accu & $\mathrm{cy}(\%)$ & Sensit & ity(\%) & Specif & ity(\%) & Accu & $\operatorname{cy}(\%)$ \\
\hline Classifier & ECG & $\mathrm{SpO}_{2}$ & ECG & $\mathrm{SpO}_{2}$ & ECG & $\mathrm{SpO}_{2}$ & ECG & $\mathrm{SpO}_{2}$ & ECG & $\mathrm{SpO}_{2}$ & ECG & $\mathrm{SpO}_{2}$ \\
\hline SVM & 0.00 & 54.00 & 100.00 & 94.10 & 75.66 & 84.36 & 66.99 & 78.18 & 72.78 & 82.84 & 71.37 & 81.70 \\
\hline C4.5 tree & 47.56 & 60.47 & 85.66 & 90.41 & 76.39 & 83.13 & 53.91 & 70.31 & 79.14 & 83.88 & 73.00 & 80.58 \\
\hline Bagging.REPTree & 45.49 & 63.73 & 92.96 & 92.40 & 81.41 & 85.43 & 69.82 & 78.23 & 80.29 & 84.25 & 77.74 & 82.79 \\
\hline Bagging.ADTree & 34.15 & 58.85 & 93.56 & 93.94 & 79.10 & 85.40 & 72.47 & 82.74 & 72.87 & 80.41 & 72.77 & 80.98 \\
\hline FT trees & 46.53 & 59.50 & 83.73 & 89.05 & 74.67 & 81.86 & 58.52 & 72.04 & 75.43 & 81.79 & 71.32 & 79.42 \\
\hline AdaBoost & 31.59 & 72.64 & 91.60 & 87.18 & 77.00 & 83.64 & 67.21 & 87.63 & 71.08 & 73.70 & 70.14 & 77.09 \\
\hline REPTree & 43.54 & 60.97 & 90.68 & 92.53 & 79.20 & 84.85 & 70.47 & 80.08 & 74.65 & 81.11 & 73.63 & 80.86 \\
\hline $\operatorname{kNN}(k=5)$ & 48.53 & 63.25 & 90.26 & 90.92 & 80.10 & 84.19 & 71.17 & 76.98 & 77.84 & 81.19 & 76.22 & 80.17 \\
\hline DecisionTable & 30.94 & 59.01 & 94.46 & 92.91 & 79.01 & 84.66 & 66.01 & 80.46 & 74.00 & 81.54 & 72.06 & 81.28 \\
\hline MLP & 30.80 & 59.17 & 93.61 & 91.71 & 78.32 & 83.79 & 61.84 & 75.68 & 70.25 & 83.25 & 68.20 & 81.41 \\
\hline
\end{tabular}

TABLE IV

Classification Results by Using ECG and $\mathrm{SpO}_{2}$ Feature Sets Together

\begin{tabular}{|c|c|c|c||c|c|c|c|c|}
\hline & \multicolumn{3}{|c||}{ Even Cost } & \multicolumn{5}{c|}{ Cost Sensitive 3 } \\
\hline & Sen. (\%) & Spe. (\%) & Acc. (\%) & Sen. (\%) & Spe. (\%) & Acc. (\%) & Training Time (s) & Testing Time (s) \\
\hline SVM & 55.20 & 94.30 & 84.74 & 79.48 & 83.13 & 82.24 & 32.706 & 2.979 \\
\hline C4.5 tree & 63.08 & 88.72 & 82.48 & 67.10 & 85.35 & 80.91 & 4.439 & 0.002 \\
\hline Bagging.REPTree & 65.64 & 93.35 & 86.60 & 79.75 & $\mathbf{8 5 . 8 9}$ & $\mathbf{8 4 . 4 0}$ & 10.399 & 0.002 \\
\hline Bagging.ADTree & 56.90 & 94.19 & 85.11 & 85.13 & 78.16 & 79.85 & 67.453 & 0.004 \\
\hline FT trees & 63.52 & 87.48 & 81.65 & 71.28 & 81.91 & 79.32 & 11.746 & 3.219 \\
\hline AdaBoost & 71.82 & 87.59 & 83.75 & $\mathbf{8 7 . 0 3}$ & 74.82 & 77.79 & 3.154 & 0.001 \\
\hline REPTree & 62.59 & 92.93 & 85.55 & 78.94 & 82.10 & 81.33 & 1.183 & 0.002 \\
\hline kNN $(k=5)$ & 63.74 & 91.03 & 84.38 & 77.69 & 82.92 & 81.65 & 0.000 & 10.559 \\
\hline DecisionTable & 58.63 & 92.96 & 84.61 & 79.75 & 81.12 & 80.79 & 14.043 & 0.005 \\
\hline MLP & 54.62 & 92.76 & 83.47 & 76.27 & 83.31 & 81.60 & 885.532 & 0.020 \\
\hline
\end{tabular}

larger summed probability is returned as the final prediction:

$$
C_{p}=\arg \max _{\{C+, C-\}}\left\{\sum_{i} P_{i}(C+), \sum_{i} P_{i}(C-)\right\} .
$$

3) Product of Probability (PP): Similar to the aforementioned approach, this scheme chooses the class with the larger product of positive/negative probabilities (can be viewed as the geometric mean criterion):

$$
C_{p}=\arg \max _{\{C+, C-\}}\left\{\prod_{i} P_{i}(C+), \prod_{i} P_{i}(C-)\right\} .
$$

4) Majority Voting $(M V)$ : The last approach chooses the class to which the majority of the classifiers agree. Let $C_{i}(C+)=1$ if classifier $i$ predicts the current epoch to be positive; otherwise, $C_{i}(C+)=0$ and apply a similar rule to $C_{i}(C-)$. Then, the final prediction is determined as

$$
C_{p}=\arg \max _{\{C+, C-\}}\left\{\sum_{i} C_{i}(C+), \sum_{i} C_{i}(C-)\right\} .
$$

In other words, it compares the numbers of classifiers which give positive/negative predictions, and picks the class with the larger number of votes.

\section{RESULTS}

\section{A. Results of Individual Classifiers}

1) Using Either ECG or $\mathrm{SpO}_{2}$ Feature Set: The classification results of using either ECG or $\mathrm{SpO}_{2}$ feature set are first tabulated in Table III. For the case of Even Cost, among all classifiers, the sensitivity, specificity, and accuracy are around
$40 \%, 90 \%$, and $70 \%$ with the ECG feature set, whereas they are $60 \%, 90 \%$, and $80 \%$ with the $\mathrm{SpO}_{2}$ feature set, respectively. To enhance the sensitivity, via testing different penalty weights, we choose a cost matrix to penalize the FN three times that of the FP (i.e., Cost Sensitive 3), at the expense of specificity but still maintaining an acceptable accuracy (around 70-80\%). Under the Cost Sensitive 3 section of Table III, the sensitivity of the ECG feature set now ranges from $53.91 \%$ to $72.47 \%$ and that of the $\mathrm{SpO}_{2}$ feature set spans over $[70.31 \%, 87.63 \%]$. The accuracies of using these two feature sets remain around $70 \%$ and $80 \%$, respectively.

2) Using Combined ECG and $\mathrm{SpO}_{2}$ Feature Set: Table IV provides both the even cost and cost-sensitive classification results based on the combined feature set. Given the Cost Sensitive 3 result in Table IV, among ten classifiers, AdaBoost with Decision Stump achieves the highest sensitivity of $87.03 \%$ but the lowest specificity (74.82\%) and accuracy (77.79\%). Bagging with REPTree enjoys both the highest specificity $(85.89 \%)$ and accuracy $(84.40 \%)$ but a relatively low sensitivity $(79.75 \%)$.

In addition, we record in Table IV the CPU time ${ }^{2}$ spent on training and testing of each classifier during the tenfold cross validation. As can be seen, MLP, Bagging with ADTree, and $S V M$ are the most computationally intensive classifiers based on the CPU time spent on training: from about 33 to $886 \mathrm{~s}$, whereas the remaining classifiers require no more than $15 \mathrm{~s}$ in training.

\footnotetext{
${ }^{2}$ The aforementioned results are obtained from a PC of a Linux system with Intel Core 2 Duo CPU E6850 @ 3.00 GHz, 2G RAM. All the classifiers are implemented in Java.
} 
TABLE V

SELECTEd FeAtures of REduced FEATURE SET FS39

\begin{tabular}{|c|c|}
\hline $\begin{array}{l}\text { Selected ECG } \\
\text { features }(8)\end{array}$ & $S C r C 2, r r f f 3, r r f f 4, r r f f 5, r r f f 6$, edrw2, edrw9, edrf5 \\
\hline $\begin{array}{l}\text { Selected } \mathrm{SpO}_{2} \\
\text { features (31) }\end{array}$ & $\begin{array}{l}\text { Svari, Smini, NumZC, Dmean, } \text { ODIS2, } \\
\text { ODIS4, ODIS3, } \\
\text { ODIS5, ODIS, ODI21, ODI23, ODI25, ODI31, ODI43, ODI45, ODI51, ODI53, } \\
\text { ODI55, odi2, odi3, odi4, tsa95, tsa90, tsa85, CTM25, } \\
\text { CTM50, CTM75, CTM100, LZCom, ApEnt }\end{array}$ \\
\hline
\end{tabular}

TABLE VI

Classification Result of Reduced Feature Set FS39 With Cost SENSITIVE 3

\begin{tabular}{|l|l|l|l|l|l|}
\hline & Sen.(\%) & Spe.(\%) & Acc. $(\%)$ & $\begin{array}{l}\text { Training } \\
\text { Time }(\mathrm{s})\end{array}$ & $\begin{array}{l}\text { Testing } \\
\text { Time }(\mathrm{s})\end{array}$ \\
\hline SVM & 78.88 & 82.57 & 81.68 & 11.162 & 0.645 \\
\hline C4.5 tree & 69.44 & 84.25 & 80.64 & 0.775 & 0.002 \\
\hline Bagging.REPTree & 79.05 & $\mathbf{8 4 . 6 2}$ & $\mathbf{8 3 . 2 6}$ & 2.184 & 0.001 \\
\hline Bagging.ADTree & 83.93 & 79.45 & 80.54 & 14.837 & 0.006 \\
\hline FT trees & 69.81 & 80.44 & 77.86 & 3.672 & 0.852 \\
\hline AdaBoost & $\mathbf{8 6 . 8 1}$ & 74.54 & 77.53 & 0.613 & 0.001 \\
\hline REPTree & 79.65 & 82.02 & 81.44 & 0.230 & 0.002 \\
\hline kNN $(k=5)$ & 78.45 & 81.77 & 80.96 & 0.000 & 2.085 \\
\hline DecisionTable & 81.11 & 80.83 & 80.90 & 2.701 & 0.002 \\
\hline MLP & 77.15 & 81.11 & 80.14 & 70.965 & 0.013 \\
\hline
\end{tabular}

\section{B. Feature Selection}

Applying the feature selection strategy described in Section II-D, 39 out of 150 features are selected as a reduced feature set (FS39) which consists of 8 ECG features and 31 $\mathrm{SpO}_{2}$ features. In particular, the eight ECG features [11] are the second-order serial correlation coefficient $(\mathrm{SCrC} 2)$, the third, fourth, fifth, and sixth discrete Fourier transform (DFT) points of RR intervals (rrff $3, r r f f 4, r r f f 5, r r f f 6)$, the spectral variances of second and ninth levels of decimated wavelet transform of EDR series (edrw2, edrw9), and the fifth DFT point of EDR series (edrf5). All the selected 39 features are listed in Table V.

Cost-sensitive classifications are carried out to assess the performance of the reduced feature set FS39 and the results are shown in Table VI. Compared with Table IV, using FS39, the computational load shrinks to around only $1 / 5$ of the one using the full combined feature set, for almost all classifiers, with less than $1 \%$ decrease in sensitivity, specificity, and accuracy. AdaBoost with Decision Stump still achieves the highest sensitivity of $86.81 \%$ while Bagging with REPTree retains the highest specificity of $84.62 \%$ and the accuracy of $83.26 \%$ among ten classifiers. In terms of computational efficiency, SVM, Bagging with ADTree, and MLP still consume the longest CPU time (11-71 s on training) while other classifiers only spend less than $3.7 \mathrm{~s}$ on training in 1 tenfold cross validation.

\section{Classifier Combination}

The result in the previous section (see Table VI) also shows that although AdaBoost with Decision Stump achieves the highest sensitivity, its specificity is the lowest among ten classifiers; Bagging with REPTree attains the highest specificity but its sensitivity is below $80 \%$. In the interest of a well-rounded classification result, we apply classifier combination proposed in Section II-E to balance the performances in both sensitivity and specificity, and hence the accuracy.
Since AdaBoost with Decision Stump and Bagging with REPTree achieve highest sensitivity and specificity, respectively, we choose them as two permanent member classifiers in our combination experiment. On the other hand, to avoid too much computation, three most computationally intensive classifiers: SVM, Bagging with ADTree, and MLP are excluded. As a result, an additional classifier is drawn from the remaining five classifiers sequentially to form a group of three experts. The reduced feature set FS39 and the $\mathrm{SpO}_{2}$ feature set are both considered in the classifier combination experiment.

Via a thorough experiment with five different choices of the third member classifier, it is found that $k N N(k=5)$ and Decision Table collaborate best with AdaBoost with Decision Stump and Bagging with REPTree. Their combination results are illustrated in Tables VII and VIII, respectively. As we can see, the sensitivity, specificity, and accuracy are all around $82 \%$ and $81 \%$ for the FS39 feature set and the $\mathrm{SpO}_{2}$ feature set, respectively, for all four combination schemes.

\section{DISCUSSION}

\section{A. Comparison Among Different Feature Sets}

Recall that in Section III-A1, if we compare the individual signals, the proposed $\mathrm{SpO}_{2}$ feature set achieves a much better performance than the ECG feature set in both even cost and cost-sensitive cases: averagely, about $25 \%$ and $12 \%$ advantage in sensitivity and $6 \%$ and $8 \%$ advantage in accuracy for Even Cost and Cost Sensitive 3, respectively. Besides, in the selected feature set FS39, the majority existence of $\mathrm{SpO}_{2}$ features $\left(31 \mathrm{SpO}_{2}\right.$ features versus 8 ECG features) also implies the higher diagnostic ability of $\mathrm{SpO}_{2}$ features than the ECG features. The reason could be explained as follows. Since the $\mathrm{SpO}_{2}$ signal is a direct reflection of the amount of oxygen that one inhales, it could directly capture the oxygen variation due to the pause/reduction of breath when sleep apnea/hypopnea happens. On the other hand, the ECG signal records the electrical activity of the heart where not only apnea/hypopnea, but also many other metabolic processes as well as, highly possibly, other heart-related disorders are taking place. In this sense, the ECG signal is more complicated and the most sleep-apnea/hypopnea-relevant information might be buried in other signals. Hence, to unveil and extract the most indicative features from ECG for SAHS detection is very challenging. Consequently, if only one sensor is allowed for SAHS detection, oximeter is preferable over ECG sensor.

Moreover, if both ECG and $\mathrm{SpO}_{2}$ signals are available, using the combined full feature set (see Table IV) can largely improve the performance of using ECG features alone (see Table III): about $15 \%, 6 \%$, and $7 \%$ increases in maximum sensitivity, specificity, and accuracy among ten classifiers, respectively. The reduced feature set FS39, obtained by our proposed cost-sensitive subset feature selection, achieves approximately the same good classification result but only requires about $1 / 5$ of the computational load of the full feature set. Comparing FS39 and the $\mathrm{SpO}_{2}$ feature set, from Tables VII and VIII, for every combination scheme, FS39 shows advantage (about 1\%) over the $\mathrm{SpO}_{2}$ feature set in sensitivity and accuracy while most of the time in specificity as well. In other words, the incorporation 
TABLE VII

Performances of a Classifier Combination With FS39 and SpO 2 Feature Sets

\begin{tabular}{|c|c|c|c|c|c|c|c|c|}
\hline Cost Sensitive 3 & \multicolumn{2}{|c|}{ Max Prob. } & \multicolumn{2}{c|}{ Average Prob. } & \multicolumn{2}{c|}{ Product Prob. } & \multicolumn{2}{c|}{ Majority Voting } \\
\hline Bagging.REPTree + AdaBoost + kNN & $\mathrm{FS} 39$ & $\mathrm{SpO}_{2}$ & $\mathrm{FS} 39$ & $\mathrm{SpO}_{2}$ & $\mathrm{FS} 39$ & $\mathrm{SpO}_{2}$ & FS39 & $\mathrm{SpO}_{2}$ \\
\hline Sensitivity (\%) & 81.87 & 81.60 & 82.41 & 81.71 & 82.19 & 81.38 & $\mathbf{8 3 . 5 5}$ & 82.41 \\
\hline Specificity (\%) & 82.07 & 81.07 & 82.03 & 81.21 & 82.16 & 81.23 & 81.25 & 80.62 \\
\hline Accuracy (\%) & 82.02 & 81.20 & 82.12 & 81.33 & 82.16 & 81.27 & 81.81 & 81.05 \\
\hline CPU Time Training (s) & 2.991 & 2.902 & 2.814 & 2.893 & 2.794 & 2.909 & 2.927 & 2.903 \\
\hline CPU Time Testing (s) & 2.291 & 1.987 & 2.115 & 1.994 & 2.074 & 1.980 & 2.262 & 1.993 \\
\hline
\end{tabular}

TABLE VIII

Performances of ANother Classifier Combination With FS39 And $\mathrm{SPO}_{2}$ Feature Sets

\begin{tabular}{|c|c|c|c|c|c|c|c|c|}
\hline Cost Sensitive 3 & \multicolumn{2}{|c|}{ Max Prob. } & \multicolumn{2}{c|}{ Average Prob. } & \multicolumn{2}{c|}{ Product Prob. } & \multicolumn{2}{c|}{ Majority Voting } \\
\hline Bagging.REPTree + AdaBoost + Decision Table & $\mathrm{FS} 39$ & $\mathrm{SpO}_{2}$ & $\mathrm{FS} 39$ & $\mathrm{SpO}_{2}$ & $\mathrm{FS} 39$ & $\mathrm{SpO}_{2}$ & $\mathrm{FS} 39_{|c|} \mathrm{SpO}_{2}$ \\
\hline Sensitivity (\%) & 82.14 & 81.82 & 82.68 & 82.41 & 82.57 & 82.25 & $\mathbf{8 3 . 6 1}$ & 82.90 \\
\hline Specificity (\%) & 81.47 & 80.69 & 81.35 & 80.48 & 81.42 & 80.57 & 80.16 & 80.43 \\
\hline Accuracy (\%) & 81.64 & 80.96 & 81.68 & 80.95 & 81.70 & 80.98 & 81.00 & 81.03 \\
\hline CPU Time Training (s) & 5.561 & 5.156 & 5.597 & 5.144 & 5.581 & 5.162 & 5.550 & 5.149 \\
\hline CPU Time Testing (s) & 0.003 & 0.004 & 0.004 & 0.005 & 0.002 & 0.003 & 0.004 & 0.004 \\
\hline
\end{tabular}

of ECG features complements $\mathrm{SpO}_{2}$ features by providing additional useful information for SAHS detection. Therefore, with access to both signals, such as in hospital, classifier combination with feature set FS39 serves as a good candidate for real-time SAHS detection. However, given a home-based setting with consideration of the cost of extra sensor, classifier combination based on the $\mathrm{SpO}_{2}$ signal alone also suffices to offer a decent performance.

\section{B. Comparison Among Different Classification Strategies}

Among the ten individual classifiers, AdaBoost with Decision Stump achieves the highest sensitivity and Bagging with REPTree attains the highest specificity and accuracy almost for all feature sets.

With classifier combination (see Tables VII and VIII), almost all three metrics improve if compared with the results of individual classifiers for both FS39 and $\mathrm{SpO}_{2}$ feature sets (Tables VI and III): the sensitivity increases at least $2 \%$ compared with Bagging with REPTree while about 7-8\% improvement in specificity and $4-5 \%$ increase in accuracy compared with AdaBoost with Decision Stump. Conclusively, better and more balanced detection results are achieved with the proposed classifier combination.

Among four different classifier combination schemes, Majority Voting always wins the highest sensitivity. Because each member classifier is already in favor of positive class due to the cost-sensitive setting, the final prediction of $M a$ jority Voting further emphasizes the sensitivity by its "hard decision" nature: based on the classes instead of the associated probabilities.

Given a real-time detection problem, the computational complexity is of concern. AdaBoost with Decision Stump and Bagging with REPTree are computationally efficient compared to other algorithms such as $S V M$ and $M L P$, in addition to their good detection performances. With the two being permanent members for classifier combination, choosing either $k N N$ or Decision
Table as the third member gives comparable classification results as shown in Tables VII and VIII. The difference mainly lies in the computational loads of training and testing. In general, the total training/testing time for the combination approach is the sum of those for individual classifiers. Note that $k N N$ is a lazy classifier that requires no time on training; thus, the total training time for the combination approach with $k N N$ stays around $2.9 \mathrm{~s}$ versus $5.3 \mathrm{~s}$ for the combination approach with Decision Table. However, since $k N N$ offloads the complexity to testing phase, the corresponding testing time of the former is about $2 \mathrm{~s}$, while the latter requires much less, around $3 \mathrm{~ms}$. As a result, the former combination is more suitable for a subjectdependent (SD) application where testing is conducted based on the model trained by the same subject's historical data. In this case, trainings need to be carried out or updated on a subject basis. The latter combination with Decision Table fits better for a subject-independent (SI) application where training is already done based on some (large) database before distributing to users who are only responsible for testing.

Regarding the memory requirement of our proposed classifier combination for real-time SAHS detection, both training and testing programs run freely within the default maximum allocated memory (455 MB) which is less than the memory size of iPhone 4 (512 MB RAM). The memory usage for the testing phase of our proposed algorithm is much less since each time only $1 \mathrm{~min}$ of data are processed. In addition, for the training process which generally requires more memory, current technology allows large amount of computations to be offloaded to servers via Internet (cloud computing) [11]. As a result, the memory issues can be effectively solved.

Finally, it is interesting to note that performances of algorithms such as $S V M$ and $M L P$ also depend on parameter settings. However, it is usually unknown beforehand which parameter setting is the best for a given problem. Locating the optimal parameter setting is often done by cross validation and grid search [36]. It is also highly probable that the best parameter setting for one database fails to cater for another database. 
Therefore, reoptimization of parameters is required for these algorithms. To avoid such tedious and database-dependent parameter optimization processes, we consider and mainly focus on some other classifiers that require few, if any, parameter adjustments, especially the algorithms chosen for our proposed combination scheme: decision-tree-based algorithms, Decision Table, and lazy classifier $k N N$. The relief from parameter optimization also potentially extends the applicability of our proposed scheme to other databases.

\section{Comparison With Other Related Works}

As mentioned before, most precedent SAHS detection approaches gave overall diagnoses on whether a suspect is with or without SAHS based on the whole overnight signals [5]-[10], [13]-[18]. Several works also developed techniques to detect SAHS on an epoch basis [8]-[12], [19]-[21]. In general, the specific classification results are database-dependent. Some works used their own clinical data [12], [15]-[18]. For instance, Heneghan et al. [12] applied linear discriminant analysis for classification with eight ECG features and five $\mathrm{SpO}_{2}$ features and obtained epoch-wise sensitivity and specificity of $51.4 \%$ and $87.3 \%$, respectively. Others [8]-[11], [20] utilized some free online databases from PhysioNet, for example, Apnea-ECG database [37] which is the database used in Computers in Cardiology 2000 Challenge of sleep apnea detection based on ECG. Two top teams [8], [9] in this challenge achieved above 90\% accuracy on an epoch basis where manual inspection was used to identify the apneic episodes in [8] while [9] also relied on manual editing to improve the performances.

Among the most recent automated real-time SAHS detection approaches, Bsoul et al. [11] employed SVM for classification based on a very comprehensive ECG feature set. A sensitivity of $96 \%$ was attained in the SD experiment while an accuracy of $86 \%$ was reported for the SI experiment based on ApneaECG database. However, as can be seen from Table III, the sensitivity, specificity, and accuracy of the SI experiment are around $67 \%, 73 \%$, and $71 \%$ using SVM with the ECG features proposed by Bsouls et al. in UCD database. Burgos et al. [20] considered data mining techniques and used eight $\mathrm{SpO}_{2}$ features for real-time SAHS detection. An accuracy of $93 \%$ is obtained on Apnea-ECG database but it contains only eight subjects' overnight $\mathrm{SpO}_{2}$ recordings. Applying Burgos' method on UCD database, only about $34 \%$ sensitivity is returned [21].

Our real-time SAHS detection is carried out on UCD database where 25 sleep-disordered-breathing suspects' ECG and $\mathrm{SpO}_{2}$ signals are both available. We investigate the diagnostic abilities of these two signals individually and in combination. According to our results, our proposed $\mathrm{SpO}_{2}$ features demonstrate a better detection ability of SAHS than the ECG features. In addition, instead of evaluating the features separately [11], [20], [21], we apply subset feature evaluation in a cost-sensitive manner for feature selection. The selected feature set FS39 is not only of high sensitivity but also of low redundancy among features. More importantly, based on investigations of ten machine-learning algorithms, we propose classifier combination to harness the advantages of individual classifiers and further improve and balance the classification performance, achieving sensitivity, specificity, and accuracy all around $82 \%$.

\section{CONCLUSION}

This paper studies the real-time SAHS detection based on ECG and $\mathrm{SpO}_{2}$ signals, both alone and in combination. According to the tenfold cross-validation result based on ten individual classifiers, our proposed $\mathrm{SpO}_{2}$ features demonstrated a better diagnostic ability than ECG features. Through a cost-sensitive subset feature selection, a reduced feature set (FS39) of 8 ECG features and $31 \mathrm{SpO}_{2}$ features is obtained. Classifier combination is proposed to balance and further improve the performance. It is found that two groups of classifier collaboration stand out among others. Classifier combination based on $\mathrm{SpO}_{2}$ features only is able to provide a satisfactory detection performance. Nevertheless, given the available ECG signal, adding certain ECG features (as those in FS39) is beneficial to the detection performance.

\section{ACKNOWLEDGMENT}

The authors thank the editor and anonymous reviewers for their constructive comments to enhance the quality and presentation of this paper. The authors would like to thank Dr. Majdi Bsoul for providing Matlab codes for [11].

\section{REFERENCES}

[1] T. Young, M. Palta, J. Dempsey, J. Skatrud, S. Weber, and S. Badr, "The occurrence of sleep-disordered breathing among middle-aged adults," New Engl. J. Med., vol. 328, no. 17, pp. 1230-1235, 1993.

[2] T. Young, L. Evans, L. Finn, and M. Palta, "Estimation of the clinically diagnosed proportion of sleep apnea syndrome in middle-aged men and women," Sleep, vol. 20, no. 9, pp. 705-706, 1997.

[3] S. Ancoli-Israel, E. R. DuHamel, C. Stepnowsky, R. Engler, M. CohenZion, and M. Marler, "The relationship between congestive heart failure, sleep apnea, and mortality in older men," Chest, vol. 124, no. 4, pp. 14001405 .

[4] W. Lee, S. Naqubadi, and M. H. Mokhlesi, "Epidemiology of obstructive sleep apnea: A population-based perspective," Expert Rev. Respir. Med., vol. 2, no. 3, pp. 349-364, Jun. 2008.

[5] U. Magalang, J. Dmochowski, S. Veeramachaneni, A. Draw, M. Mador, A. El-Solh, and B. Grant, "Prediction of the apnea-hypopnea index from overnight pulse oximetry," Chest, vol. 124, no. 5, pp. 1694-1701, 2003.

[6] N. Netzer, R. Stoohs, C. Netzer, K. Clark, and K. Strohl, "Using the Berlin Questionnaire to identify patients at risk for the sleep apnea syndrome," Ann. Int. Med., vol. 131, no. 7, pp. 485-491, 1999.

[7] A. Ng, T. Koh, E. Baey, and K. Puvanendran, "Speech-like analysis of snore signals for the detection of obstructive sleep apnea," in Proc. Int. Conf. Biomed. Pharmaceut. Eng., 2006, pp. 99-103.

[8] J. McNames and A. Fraser, "Obstructive sleep apnea classification based on spectrogram patterns in the electrocardiogram," in Proc. Comput. Cardiol., 2000, pp. 749-752.

[9] B. Raymond, R. Cayton, R. Bates, and M. Chappell, "Screening for obstructive sleep apnoea based on the electrocardiogram-The computers in cardiology challenge," in Proc. Comput. Cardiol., 2000, pp. 267-270.

[10] Z. Shinar, A. Baharav, and S. Akselrod, "Obstructive sleep apnea detection based on electrocardiogram analysis," in Proc. Comput. Cardiol., 2000, pp. 757-760.

[11] M. Bsoul, H. Minn, and L. Tamil, "Apnea MedAssist: Real-time sleep apnea monitor using single-lead ECG," IEEE Trans. Inf. Technol. Biomed., vol. 15 , no. 3 , pp. 416-427, May 2011.

[12] C. Heneghan, C. Chua, J. Garvey, P. De Chazal, R. Shouldice, P. Boyle, and W. McNicholas, "A portable automated assessment tool for sleep apnea using a combined Holter-oximeter," Sleep, vol. 31, no. 10, pp. 1432-1439, 2008 . 
[13] P. Lévy, J. Pépin, C. Deschaux-Blanc, B. Paramelle, and C. Brambilla, "Accuracy of oximetry for detection of respiratory disturbances in sleep apnea syndrome," Chest, vol. 109, no. 2, pp. 395-399, 1996.

[14] L. Olson, A. Ambrogetti, and S. Gyulay, "Prediction of sleep-disordered breathing by unattended overnight oximetry," J. Sleep Res., vol. 8, no. 1, pp. $51-55,1999$.

[15] C. Lin, C. Yeh, C. Yen, W. Hsu, and L. W. Hang, "Comparison of the indices of oxyhemoglobin saturation by pulse oximetry in obstructive sleep apnea hypopnea syndrome," Chest, vol. 135, no. 1, pp. 86-93, 2008.

[16] R. Hornero, D. Alvarez, D. Abasolo, F. del Campo, and C. Zamarron, "Utility of approximate entropy from overnight pulse oximetry data in the diagnosis of the obstructive sleep apnea syndrome," IEEE Trans. Biomed. Eng., vol. 54, no. 1, pp. 107-113, Jan. 2007.

[17] D. Alvarez, R. Hornero, D. Abásolo, F. Campo, and C. Zamarrón, "Nonlinear characteristics of blood oxygen saturation from nocturnal oximetry for obstructive sleep apnoea detection," Physiol. Meas., vol. 27, pp. 399-412, 2006

[18] C. Zamarrón, F. Gude, J. Barcala, J. Rodriguez, and P. Romero, "Utility of oxygen saturation and heart rate spectral analysis obtained from pulse oximetric recordings in the diagnosis of sleep apnea syndrome," Chest, vol. 123, no. 5, pp. 1567-1576, 2003.

[19] N. Oliver and F. Flores-Mangas, "HealthGear: A real-time wearable system for monitoring and analyzing physiological signals," in Proc. Body Sensor Netw., 2006, pp. 61-64.

[20] A. Burgos, A. Goñi, A. Illarramendi, and J. Bermudez, "Real-time detection of apneas on a PDA," IEEE Trans. Inf. Technol. Biomed., vol. 14, no. 4, pp. 995-1002, Jul. 2010.

[21] B. Xie, W. Qiu, H. Minn, L. Tamil, and M. Nourani, "An improved approach for real-time detection of sleep apnea," in Proc. Int. Conf. BioInspired Syst. Signal Process., 2011, pp. 169-175.

[22] St. Vincent's University Hospital/University College Dublin Sleep Apnea Database. (2008). [Online]. Available: http://www.physionet.org/pn3/ ucddb/

[23] A. L. Goldberger, L. A. N. Amaral, L. Glass, J. M. Hausdorff, P. C. Ivanov, R. G. Mark, J. E. Mietus, G. B. Moody, C.-K. Peng, and H. E. Stanley, "PhysioBank, PhysioToolkit, and PhysioNet: Components of a new research resource for complex physiologic signals," Circulation, vol. 101, no. 23, pp. e215-e220, 2000 .

[24] M. Hall, E. Frank, G. Holmes, B. Pfahringer, P. Reutemann, and I. Witten, "The WEKA data mining software: An update," ACM SIGKDD Explorat. Newslett., vol. 11, no. 1, pp. 10-18, 2009.

[25] C.-C. Chang and C.-J. Lin. (2001). LIBSVM: A library for support vector machines [Online]. Available: http://www.csie.ntu.edu.tw/ cjlin/libsvm

[26] D. Aha and D. Kibler, "Instance-based learning algorithms," Mach. Learn., vol. 6, pp. 37-66, 1991.

[27] R. Kohavi, "The power of decision tables," in Proc. 8th Eur. Conf. Machine Learning, 1995, pp. 174-189.

[28] T. M. Mitchell, Machine Learning (McGraw Hill Series in Computer Science). New York: McGraw-Hill, 1997.

[29] R. Quinlan, C4.5: Programs for Machine Learning. San Mateo, CA: Morgan Kaufmann, 1993

[30] N. Landwehr, M. Hall, and E. Frank. (2005, May). Logistic model trees. Mach. Learn. [Online]. 59, pp. 161-205. Available: http://dx.doi.org/10.1007/s10994-005-0466-3

[31] Y. Freund and R. E. Schapire, "Experiments with a new boosting algorithm," in Proc. 13th Int. Conf. Mach. Learn., 1996, pp. 148-156.

[32] L. Breiman, "Bagging predictors," Mach. Learn., vol. 24, no. 2, pp. $123-$ 140, 1996.
[33] Y. Freund, "The alternating decision tree learning algorithm," in Proc. 6th Int. Conf. Mach. Learn., 1999, pp. 124-133.

[34] M. A. Hall, "Correlation-based feature subset selection for machine learning" Ph.D. dissertation, Univ. Waikato, Hamilton, New Zealand, 1998.

[35] S. Tulyakov, S. Jaeger, V. Govindaraju, and D. Doermann, Review of Classifier Combination Methods. vol. 90. Berlin/Heidelberg, Germany: Springer-Verlag, 2008, pp. 361-386.

[36] C.-W. Hsu, C.-C. Chang, and C.-J. Lin. (2010). "A practical guide to support vector classification," [Online]. Available: http://www csie.ntu.edu.tw/ cjlin/libsvm

[37] "CinC Challenge 2000 data sets: Data for development and evaluation of ECG-based apnea detectors," (2000). [Online]. Available: http:// www.physionet.org/physiobank/database/apnea-ecg/

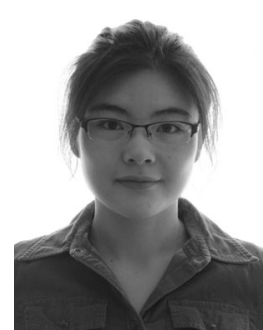

Baile Xie (S'11) received the B.S. degree in communications engineering from the University of Electronic Science and Technology of China, Chengdu, China, in 2008.

She is currently working toward the Ph.D. degree in electrical engineering at the University of Texas at Dallas, Richardson. Her research interests include wireless communications, signal processing, and wireless health-care technologies.

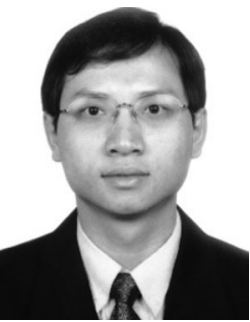

Hlaing Minn (S'99-M'01-SM'07) received the B.E. degree in electronics from the Yangon Institute of Technology, Yangon, Myanmar, in 1995, the M.Eng. degree in telecommunications from the Asian Institute of Technology, Pathumthani, Thailand, in 1997 and the $\mathrm{Ph} . \mathrm{D}$. degree in electrical engineering from the University of Victoria, Victoria, BC, Canada, in 2001.

He was a Postdoctoral Fellow at the University of Victoria from January to August of 2002 In September 2003, he joined the Erik Jonsson School of Engineering and Computer Science, University of Texas at Dallas, Richardson, where he is currently an Associate Professor. His research interests include wireless communications, statistical signal processing, error control, detection, estimation, synchronization, signal design, cross-layer design, cognitive radios, and wireless health-care applications.

Dr. Minn is an Editor for the IEEE TRANSACTIONS ON COMMUNICATIONS and International Journal of Communications and Networks. He served as a Co-Chair of the Wireless Access Track in the IEEE VTC 2009 (Fall) and as a member of technical program committee in several IEEE major conferences. 